\title{
AN OPTICAL STUDY OF THE STAR FORMATION REGION NGC 7129
}

\author{
A.I. Gomez de Castro and C. Eiroa \\ Observatorio Astronómico Nacional, Spain
}

NGC 7129 is a region where many signs of active star formation have been observed. Three water vapor masers, five Herbig-Haro objects, two FIR sources, two molecular outflows and several PMS stars have been detected. We have obtained very deep CCD images of the GGD nebulosities found in the region. The observations have been carried out with the $3.5 \mathrm{~m}$. telescope at the Calar Alto Observatory (Almeria, Spain). The main conclusions inferred from the data are: (1) The Herbig-Haro nature of GGD 32, GGD 34, GGD 35, HH 103 and HH 105 is confirmed, however GGD 33 has shown to be a PMS star, GGD 33a, with a cometary nebulosity, GGD 33b; (2) GGD 32 and HH 103 are embedded in an extended structure (at least as large as $0.465 \times 1.36 \mathrm{pc}$ ), where another Herbig-Haro object and an arc connecting GGD 32 and $\mathrm{HH} 103$ have been detected. The whole ensemble seems to be linked to a third molecular outflow which is not well resolved in the available radiomaps; (3) The arc connecting GGD 32 and HH 103 contains low excitation material and probably traces a low density cavity within the molecular cloud; (4) GGD 34 is constituted by three condensations aligned in the East-West direction, the western one is a bow-shock where the cooling region is resolved and has a size of around $2000 \mathrm{AU} ;(5)$ the PMS star associated to GGD 35 has been identified. The mass outflow is highly collimated close to the star, but at a distance of $0.072 \mathrm{pc}$ from the star it is deflected by an external agent which deviates it in the direction of the molecular outflow associated to LkHo 234.

* A.I. Gomez de Castro is supported by Posdoc fellowship of the M.E.C.

\section{STAR FORMATION IN THE NGC 2071 MOLECULAR CLOUD}

\author{
N.J. Evans \\ Departtment of Astronomy, University of Texas
}

The NGC 2071 molecular cloud has been studied with a broad array of techniques, including a large scale study of CS emission, high resolution scans in the far-infrared, $\mathrm{NH}_{3}$ studies with the VLA, and near-infrarred imaging. The far-infrared emission constrains the density distribution to fall off approximately as $\mathrm{r}^{-1}$. The $\mathrm{NH}_{3}$ data strongly supports the presence of a disk oriented perpendicular to the molecular outflow, while the CS emission indicates the presence of dense gas in the region. The results will be combined into a coherent picture of this region of current star formation and molecular outflow. 